\title{
Robust Optimization of Storage Investment on Transmission Networks
}

\author{
Rabih A. Jabr, Senior Member, IEEE, Izudin Džafić, Senior Member, IEEE, \\ and Bikash C. Pal, Fellow, IEEE
}

\begin{abstract}
This paper discusses the need for the integration of storage systems on transmission networks having renewable sources, and presents a tool for energy storage planning. The tool employs robust optimization to minimize the investment in storage units that guarantee a feasible system operation, without load or renewable power curtailment, for all scenarios in the convex hull of a discrete uncertainty set; it is termed ROSION - Robust Optimization of Storage Investment On Networks. The computational engine in ROSION is a specific tailored implementation of a column-and-constraint generation algorithm for two-stage robust optimization problems, where a lower and an upper bound on the optimal objective function value are successively calculated until convergence. The lower bound is computed using mixed-integer linear programming and the upper bound via linear programming applied to a sequence of similar problems. ROSION is demonstrated for storage planning on the IEEE 14-bus and 118-bus networks, and the robustness of the designs is validated via Monte-Carlo simulation.
\end{abstract}

Index Terms-Design optimization, energy storage, integer linear programming, optimization methods, power system planning.

\section{NOMENCLATURE}

\section{A. Storage Planning Problem}

$B_{i j} \quad$ Line susceptance between nodes $i$ and $j$, defined as a positive number.

$c_{i 0} \quad$ Fixed cost of the generator at node $i$.

$c_{i k} \quad$ Slope of line segment $k$ of the generator cost curve at node $i$.

$E_{i 0} \quad$ Initial stored energy in the storage unit at node $i$.

$E_{i f} \quad$ Final stored energy in the storage unit at node $i$.

$E_{i}^{\max } \quad$ Maximum storage capacity of the unit at node $i$.

$E_{i}^{\text {min }} \quad$ Minimum storage capacity of the unit at node $i$.

$f_{i}($.$) \quad Convex generation cost function at node i$.

$K_{D} \quad$ Penalty coefficient for demand curtailment.

$K_{R} \quad$ Penalty coefficient for renewable energy curtailment.

$K_{S} \quad$ Capital recovery factor of storage investment.

$n \quad$ Number of nodes in the system.

$N_{i} \quad$ Number of linear segments in the cost curve of the generator at node $i$.

This work was supported by the American University of Beirut Research Board.

R. A. Jabr is with the Department of Electrical \& Computer Engineering, American University of Beirut, P.O. Box 11-0236, Riad El- Solh / Beirut 1107 2020, Lebanon (email: rabih.jabr@aub.edu.lb).

I. Džafić is with the International University of Sarajevo, Hrasnička cesta 15, 71210 Sarajevo, Bosnia (email: idzafic@ieee.org).

B. C. Pal is with the Electrical and Electronic Engineering Department, Imperial College, London SW7 2AZ, U.K. (e-mail: b.pal@imperial.ac.uk).
$P_{C D i}(t)$ Demand power curtailment at node $i$ and in period $t$.

$P_{C R i}(t)$ Renewable power curtailment at node $i$ and in period $t$.

$P_{D i}(t) \quad$ Power demand at node $i$ and in period $t$.

$P_{\text {Gik }}(t)$ Power generation at node $i$, over segment $k$, and in period $t$.

$P_{G i}(t) \quad$ Power generation at node $i$ and in period $t$.

$P_{G i}^{(k)} \quad$ Maximum power generation at node $i$ and over segment $k$.

$P_{G i}^{\min } \quad$ Minimum power generation at node $i$ (equal to $\left.P_{G i}^{(0)}\right)$.

$P_{i j}^{\max } \quad$ Maximum power flow limit between nodes $i$ and $j$.

$P_{R i}(t) \quad$ Renewable power generation at node $i$ and in period $t$.

$P_{S i}^{c(\max )}$ Maximum hourly charging power rate of the storage unit at node $i$.

$P_{S i}^{c}(t) \quad$ Power charge into the storage unit at node $i$ and in period $t$.

$P_{S i}^{d(\max )}$ Maximum hourly discharging power rate of the storage unit at node $i$.

$P_{S i}^{d}(t) \quad$ Power discharge from the storage unit at node $i$ and in period $t$.

$R R_{i}^{\max }$ Maximum hourly up/down ramp-rate limit of the generator at node $i$.

$S C_{i} \quad$ Storage investment cost at node $i$.

$\alpha_{i} \quad$ Binary variable equal to 1 if storage is installed at node $i$ and equal to zero otherwise.

$\alpha_{i}^{c}(t) \quad$ Binary variable equal to 1 if the storage installed at node $i$ is being charged in period $t$, and equal to zero otherwise.

$\alpha_{i}^{d}(t) \quad$ Binary variable equal to 1 if the storage installed at node $i$ is being discharged in period $t$, and equal to zero otherwise.

$\eta_{c} \quad$ Storage efficiency during charging.

$\eta_{d} \quad$ Storage efficiency during discharging.

$\Gamma_{c} \quad$ Storage budget expressed in terms of the allowed investment cost.

$\Gamma_{n} \quad$ Storage budget expressed in terms of the allowed number of units.

$\Omega(i) \quad$ Set of nodes connected to noode $i$ by a branch.

$\theta_{i j}(t) \quad$ Difference between nodal angles $i$ and $j$ in period $t$. 


\section{B. ROSION}

$m$

$u^{(s)}$

$U$

$x$

$y$

$y^{(s)}$

$\Theta$
Number of scenarios in the discrete uncertainty set.

Scenario $s$ in the discrete uncertainty set.

Discrete uncertainty set.

Vector of first stage (design decision or unadjustable) variables.

Vector of second stage (control decision or adjustable) variables.

Vector of second stage variables corresponding to scenario $s$.

Set of indices of the scenarios that are generated by the slave problem.

\section{INTRODUCTION}

The proliferation of renewable energy in power grids necessitates the increased use of conventional generation reserve to maintain power balance. While some systems have the capacity to provide sufficient reserve to mitigate the renewable power intermittency as part of their ancillary services, others lack flexibility in their generation portfolios [1]. Energy storage systems can buffer the output of intermittent renewable sources and consequently contribute to frequency regulation, system stability, peak shaving, and deferral of transmission line investment; the storage systems comprise batteries, flywheels, pumped hydroelectric, or compressed air technology. The need for investment in storage is most pronounced in systems having high renewable penetration [2], or in networks that have renewable energy integration and limited ramping capacity [1]. In such networks, energy storage eliminates the need for renewable power and load curtailment by allowing the generation and consumption of power to occur non-concurrently.

Several of the storage planning tools are based on a multiperiod dynamic Optimal Power Flow (OPF) formulation, where the OPF solution in consecutive time intervals is constrained by the available storage capacity, the ramp rates of conventional generators, and the charge/discharge rates of storage units. Even if the cost of storage is not explicitly accounted for in the multi-period simulation, the activity of the storage devices in the optimal policy gives guidance on their required rated energy, power, and location. For a system with a single generator and a single load, Ref. [3] demonstrates that the optimal policy calls for generating more than the demand to charge the battery, and then generating less than the demand so that the battery complements the conventional generation in supplying the load. In the general case that accounts for several generators and loads, energy storage is shown to increase the availability of renewable energy on the network [4]. Ref. [5] extends the dynamic OPF by using chance constraints to account for Gaussian distributed errors in the simulated wind energy production and load profile. Stochastic formulations for the storage investment problem have been recently discussed [6], [7]; Ref. [6] employed a linear program with a limited number of scenarios having pre-assigned probability values, whereas [7] used a two-point estimate probabilistic OPF combined with a genetic algorithm to maximize wind power utilization. The multi-period OPF formulations, as employed in [3]-[7], require a constant power assumption over each time interval and implicitly assume that power variations within the time interval are balanced via additional regulation services. The effect of such regulation services on storage investment has been studied for the case where the regulation control is carried out via local generation [2] and battery storage systems [8].

While most of the literature on storage planning used a DC network model [2], [4]-[7], there have been some notable contributions that use an AC model and account for voltage and reactive power in their solutions [9]-[13]. Ref. [9] demonstrated a dynamic AC-OPF planning solution for a network in Ontario, where wind energy is stored at night and released during the day. The approach in [9] was extended in [10] to account for the reactive power of distributed generation and battery systems. Both [9] and [10] require the specification of charge and discharge periods as parts of the input data; this requirement has been removed in [11], but at the expense of solving a sequence of nonlinear programming problems where each has a different setting of the charge and discharge periods. The use of an AC model leads to increased accuracy in the planning. However, the resulting nonlinear programming model may exhibit convergence problems as the nonlinearity appears in all intervals; moreover, there is no guarantee that the solution is global. Refs. [12], [13] proposed a Semi Definite Programming (SDP) relaxation of the multi-period storage planning problem with an AC model. The solution to the SDP relaxation is globally optimal and feasible to the planning problem provided that the duality gap is zero - a condition that is likely to hold in practice but cannot be guaranteed prior to solving the planning problem. In addition, the SDP solution does not scale well with the problem size. The use of the AC model therefore requires further research and development for general use in a planning tool.

This research proposes a novel tool called ROSION for robust investment planning of storage systems in networks having renewable energy sources. ROSION produces a minimum cost plan of storage device locations together with their power and energy ratings such that a feasible solution (without load/renewable energy curtailment) is guaranteed to exist for any load and renewable energy scenario in a predefined uncertainty set. The uncertainty set is the convex hull of several points that constitute scenarios selected by the planner. The use of scenarios to define robust optimization problems has been discussed in [14], [15]. The scenarios are themselves referred to as the discrete uncertainty set and are chosen to represent extreme operating cases that the system is likely to encounter over the planning horizon. In power system planning applications, the scenarios are primarily obtained from load forecasts, which are practically based on load demand and econometric models that use historical data, together with economic forecasts and inputs from government sources [16]. Utilities perform annual long-term load forecasting on a yearly basis for the purpose of planning and investment [16], [17]. In fact, the importance of load-growth models for long-term planning has been already discussed in the technical literature. Ref. [18] presented a Markov demand model that considers a constant continuous deviation, with zero mean, around a 
predictable trend and the possibility of a sudden load increase because of an industrial plant moving in; the parameters of the Markov model can be estimated from past experience and economic prediction. An example in [18] shows the prediction of the minimum and maximum bus load values, based on which scenarios can be constructed; ref. [19], which discusses a recent long-term expansion planning study, quotes minimum and maximum bus load values that reflect conservative and highly uncertain demand estimates.

Unlike previous work on energy storage planning, the proposed problem formulation considers that storage devices practically have discrete sizes and power ratings. It also guarantees that for any scenario chosen from the uncertainty set, there exists a charge/discharge pattern free of simultaneous charging and discharging; the conditions for computing the charge/discharge patterns are much more flexible than what was proposed in previous work [9]-[11]. ROSION implements a solution to a two-stage robust optimization formulation using a distinct implementation of the column-and-constraint generation algorithm [14]. The need for robust optimization solutions has been recognized in several application areas [15], [19][23]. It is also preferred in storage investment planning over stochastic optimization, where practical applications require accurate statistical models that are not usually available [24].

The rest of this paper is organized as follows. Section II presents a mathematical formulation of the storage-planning model including constraints on the network, generation and storage rate limits, storage capacities, and storage dynamics that rule out simultaneous charging and discharging. The solution engine behind ROSION is detailed in section III. Numerical results that include planning solutions are given in section IV and validated through Monte-Carlo simulation; the computational behavior is also discussed. Section V concludes the paper.

\section{Energy Storage Planning}

Consider a power network having $n$ nodes with demand $\left(P_{D i}(t)\right)$, dispatchable generation $\left(P_{G i}(t)\right)$, and renewable energy sources $\left(P_{R i}(t)\right)$ connected to them. Storage is defined in accordance with the generator convention, i.e. the storage power is positive during discharge periods $\left(P_{S i}(t)=P_{S i}^{d}(t)\right)$ and negative during charge periods $\left(P_{S i}(t)=-P_{S i}^{c}(t)\right)$. In all cases, the index $i$ runs from 1 to $n$ with the understanding that any quantity (such as dispatchable generation) that is not connected to a node is set to zero. The objective of storage planning, for a single scenario of load and renewable generation profiles over times $t=1, \ldots, 24 \mathrm{~h}$, comprises the daily cost of operating conventional generation, the storage investment cost per interest period (one year or 365 days), and penalty terms for demand and renewable power curtailment [6]:

$$
\begin{array}{r}
\psi=\sum_{t=1}^{24} \sum_{i=1}^{n} \sum_{k=1}^{N_{i}}\left(c_{i k} P_{G i k}(t)+c_{i 0}\right)+\frac{K_{S}}{365} \sum_{i=1}^{n} \alpha_{i} S C_{i} \\
+K_{D} \sum_{t=1}^{24} \sum_{i=1}^{n} P_{C D i}(t)+K_{R} \sum_{t=1}^{24} \sum_{i=1}^{n} P_{C R i}(t)
\end{array}
$$

where the storage investment cost at bus $i\left(S C_{i}\right)$ is in function of the rated power and energy of the device, and $\alpha_{i}$ is a binary variable which takes the value one if storage is installed at node $i$ and zero otherwise:

$$
\alpha_{i} \in\{0,1\}, i=1, \ldots, n
$$

A storage investment budget, expressed in terms of the allowed cost (3) or the number of allowed storage units (4), can be also included:

$$
\begin{gathered}
\sum_{i=1}^{n} \alpha_{i} S C_{i} \leq \Gamma_{c} \\
\sum_{i=1}^{n} \alpha_{i} \leq \Gamma_{n}
\end{gathered}
$$

The physical and technical constraints that govern the planning problem are listed below:

- Generator dispatch and ramp-rate limits

$$
\begin{gathered}
P_{G i}(t)=P_{G i}^{\min }+\sum_{k=1}^{N_{i}} P_{G i k}(t), i=1, \ldots n, t=1, \ldots 24 \\
0 \leq P_{G i k}(t) \leq P_{G i}^{(k)}-P_{G i}^{(k-1)} \\
i=1, \ldots n, k=1, \ldots, N_{i}, t=1, \ldots 24 \\
-R R_{i}^{\text {max }} \leq P_{G i}(t)-P_{G i}(t-1) \leq R R_{i}^{\max } \\
i=1, \ldots n, t=2, \ldots, 24
\end{gathered}
$$

- Limits on load and renewable generation curtailment

$$
\begin{aligned}
& 0 \leq P_{C D i}(t) \leq P_{D i}(t), i=1, \ldots, n, t=1, \ldots, 24 \\
& 0 \leq P_{C R i}(t) \leq P_{R i}(t), i=1, \ldots, n, t=1, \ldots, 24
\end{aligned}
$$

- DC power flow equations and line flow limits

$$
\begin{array}{r}
P_{G i}(t)+P_{S i}^{d}(t)-P_{S i}^{c}(t)+P_{C D i}(t)-P_{C R i}(t) \\
-\sum_{j \in \Omega(i)} B_{i j} \theta_{i j}(t)=P_{D i}(t)-P_{R i}(t) \\
i=1, \ldots n, t=1, \ldots, 24 \\
-P_{i j}^{\max } \leq B_{i j} \theta_{i j}(t) \leq P_{i j}^{\max }, j \in \Omega(i) \\
i=1, \ldots n, t=1, \ldots, 24
\end{array}
$$

- Storage dynamics, capacity, and charge/discharge rate limits

$$
\begin{gathered}
\left(E_{i}^{\text {min }}-E_{i 0}\right) \alpha_{i} \leq \sum_{\tau=1}^{t}\left(\eta_{c} P_{S i}^{c}(\tau)-\frac{1}{\eta_{d}} P_{S i}^{d}(\tau)\right) \\
\leq\left(E_{i}^{\text {max }}-E_{i 0}\right) \alpha_{i} \\
i=1, \ldots, n, t=1, \ldots, 23 \\
\left(E_{i f}-E_{i 0}\right) \alpha_{i} \leq \sum_{\tau=1}^{24}\left(\eta_{c} P_{S i}^{c}(\tau)-\frac{1}{\eta_{d}} P_{S i}^{d}(\tau)\right) \\
\leq\left(E_{i f}-E_{i 0}\right) \alpha_{i} \\
i=1, \ldots, n \\
0 \leq P_{S i}^{c}(t) \leq P_{S i}^{c(\max )} \alpha_{i}^{c}(t), i=1, \ldots, n, t=1, \ldots, 24 \\
0 \leq P_{S i}^{d}(t) \leq P_{S i}^{d(\max )} \alpha_{i}^{d}(t), i=1, \ldots, n, t=1, \ldots, 24 \\
\alpha_{i}^{c}(t)+\alpha_{i}^{d}(t) \leq \alpha_{i}, i=1, \ldots, n, t=1, \ldots, 24
\end{gathered}
$$




$$
\alpha_{i}^{c}(t) \in\{0,1\}, \alpha_{i}^{d}(t) \in\{0,1\}, i=1, \ldots, n, t=1, \ldots, 24
$$

Equation (5) expresses the conventional generation power in function of its interval components $P_{G i k}(t)$, where each is bounded by the limits in (6); the corresponding piecewise linear generator cost curve in (1) is assumed to be convex, which guarantees the validity of the piecewise linear formulation based on separable programming [25]. Consider for illustration Fig. 1 which shows a convex generation cost function $f_{i}\left(P_{G i}(t)\right)$. Let $P_{G i}^{(k)}$ denote the $k^{t h}\left(k=0, \ldots, N_{i}\right)$ breaking point of $f_{i}\left(P_{G i}(t)\right)$ with $P_{G i}^{(0)}=P_{G i}^{\min }$ and $P_{G i}^{\left(N_{i}\right)}=P_{G i}^{\max }$; the coefficients $c_{i k}$ in (1) are given by:

$$
\begin{gathered}
c_{i k}=\frac{f_{i}\left(P_{G i}^{(k)}\right)-f_{i}\left(P_{G i}^{(k-1)}\right)}{P_{G i}^{(k)}-P_{G i}^{(k-1)}}, i=1, \ldots n, k=1, \ldots, N_{i} \\
c_{i 0}=f_{i}\left(P_{G i}^{(0)}\right)=f_{i}\left(P_{G i}^{\min }\right), i=1, \ldots n
\end{gathered}
$$

The fact that $f_{i}\left(P_{G i}(t)\right)$ is a convex function ensures that $c_{i 1}<c_{i 2}<\ldots<c_{i N_{i}}$. This implies that in the optimization, the variable $P_{G i j}(t)$ is more attractive than $P_{G i k}(t)$ for $j<k$, and will always take a nonzero value before $P_{G i k}(t)$. The generation ramp-rate limits, which constrain the maximum allowable change between two consecutive intervals, are given in (7). The values of the curtailed demand (8) and renewable generation (9) are set to less than the actual values of the quantities. The power network is modeled by a DC power flow (10) in each time period, where the power flow constraints (11) are expressed in terms of the nodal angles. The storage dynamics are given by (12) and (13). Equation (12) accounts for the initial stored energy, the minimum and maximum storage limits, and the charge/discharge storage efficiencies; (13) is the same as (12) except that it allows setting a final value for the stored energy. Both (12) and (13) are included in the formulation when storage is installed at bus $i\left(\alpha_{i}=1\right)$, and vanish from the constraint set otherwise $\left(\alpha_{i}=0\right)$. The maximum rate for charging and discharging storage units is constrained using (14) and (15), and (16)-(17) guarantee that there will be no simultaneous charging and discharging. Simultaneous charging and discharging is unrealistic for most storage technologies. Given that the storage roundtrip efficiency is less than $100 \%$, coexistent charging and discharging may occur in the absence of (16)-(17); an example is shedding renewable energy by dissipating it through the storage unit.

The Mixed-Integer Linear Programming (MILP) problem, given by (1)-(17), can be written in matrix form [23]:

$$
\min c^{T} x+\gamma
$$

subject to

$$
\begin{gathered}
\gamma \geq d^{T} y \\
A x \leq b, x \in\{0,1\} \\
G y \leq h \\
T x+Q y \leq r \\
I_{u} y=u
\end{gathered}
$$

The vector $x$ includes all binary variables and the vector $y$ all continuous variables. In matrix form, the objective

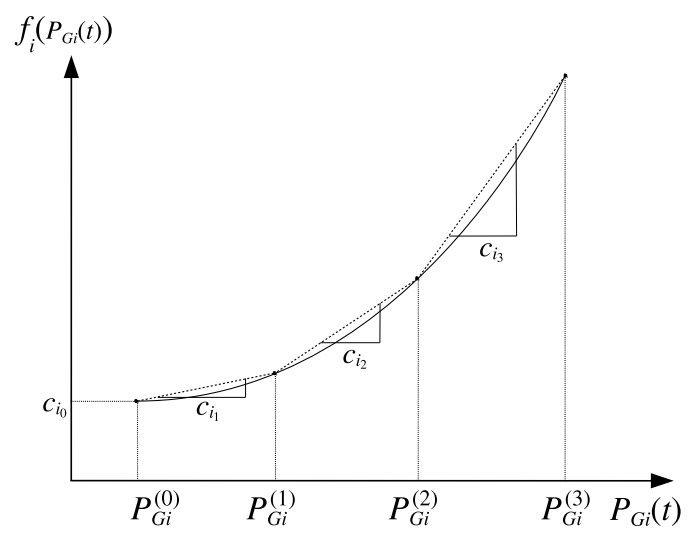

Fig. 1. Convex generation cost function

function (1) is split into two parts: (20) and (21). The storage budget $((3)$ or $(4))$, the constraints that preclude simultaneous charging/discharging (16), and the specification of binary variables ((2) and (17)) are grouped in (22). Equation (23) collects all constraints of the planning problem that involve only continuous variables ((5)-(11)), whereas (24) accounts for constraints with both binary and continuous variables ((12)(15)). Equation (25) selects the components of $y$ that are equal to the values of demand and renewable generation grouped in vector $u$; these values are considered as uncertain in the robust optimization formulation in Section III.

\section{ROSION}

The vector $u$ on the right had side of (25) represents uncertain or noisy input data. In this respect, the variables can be grouped into two sets: (i) $x$ which denotes the vector of first stage binary decision variables whose optimal value is not subject to adjustment after the realization of the uncertain input parameters; the variables in $x$ define the storage investment plan and are referred to as the design or unadjustable decision variables of the "here-and-now" decisions. (ii) $y$ which denotes the vector of second stage continuous (control decision) variables whose optimal value is conditioned on the realization of the uncertain parameters and the optimal value of the design variables. The vector $y$ comprises the variables of generation dispatch, the storage charge/discharge power, the amount of curtailed load/renewable generation, the bus voltage angles, and the uncertain parameter values given by (25); they are the adjustable decision variables of the "wait-and-see" decisions. If the vector of unadjustable binary variables is known, then the adjustable variables can be computed for any realization of the uncertain parameters using a linear program ((20)-(25) with fixed binary variables). This linear program, also known as the second-stage problem, is feasible for any realization of the uncertain parameters because the underlying model has terms that penalize the curtailment of demand and and renewable power. In two-stage robust optimization terminology, the second-stage problem displays relatively complete recourse [14].

When the planning problem is deterministic, as in (20)-(25), different realizations of the uncertain parameters may lead 
to wait-and-see decisions that result in power curtailment or costly adjustments of balancing reserves. This can be mitigated by solving a two-stage robust optimization formulation that hedges against the uncertainty of the input data. Consider a scenario-based description of the uncertain data [14], [15]: $u \in U=\left\{u^{(1)}, \ldots, u^{(m)}\right\}$, and let the corresponding adjustable decision variables belong to the set $\left\{y^{(1)}, \ldots, y^{(m)}\right\}$. The two-stage robust optimization formulation can be written as follows:

$$
\min c^{T} x+\gamma
$$

subject to

$$
\begin{gathered}
\gamma \geq d^{T} y^{(s)}, s=1, \ldots, m \\
A x \leq b, x \in\{0,1\} \\
G y^{(s)} \leq h, s=1, \ldots m \\
T x+Q y^{(s)} \leq r, s=1, \ldots, m \\
I_{u} y^{(s)}=u^{(s)}, s=1, \ldots, m
\end{gathered}
$$

The two-stage robust optimization formulation practically includes a relatively large number of scenarios, and therefore leads to a large-scale Mixed-Integer Linear Programming (MILP) problem (26)-(31) which is hard to solve. The columnand-constraint generation (C\&CG) algorithm solves the MILP problem (26)-(31) by successively computing stronger lower bounds of the optimal objective function value [14]. The lower bounds are calculated from MILP problems that are formed using a subset of scenarios in $U$ and are therefore valid relaxations of the two-stage robust optimization problem; each successive iteration involves considering an additional nontrivial scenario. The $\mathrm{C} \& \mathrm{CG}$ algorithm operates in a masterslave framework: the master problem computes $x$ and a corresponding lower bound for a subset of scenarios, and the slave problem finds the corresponding upper bound with $x$ fixed over all scenarios in $U$. The algorithm terminates when the upper and lower bounds are equal within a prespecified tolerance $\epsilon$, otherwise the upper bound computation serves to identify a scenario that should be considered in the next solution of the master problem. To present the details of the C\&CG algorithm, the the two-stage robust optimization problem (26)-(31) is rewritten in compact matrix form:

$$
\min _{x}\left[c^{T} x+\max _{u \in U} \min _{y \in F(x, u)} d^{T} y\right]
$$

subject to

$$
A x \leq b, x \in\{0,1\}
$$

where

$$
F(x, u)=\left\{y: G y \leq h, Q y \leq r-T x, I_{u} y=u\right\}
$$

The corresponding slave (or second-stage) problem is defined with fixed $x$ :

$$
\max _{u \in U} \min _{y \in F(x, u)} d^{T} y
$$

ROSION implements the following C\&CG algorithm:
1) Initialize $L B=-\infty, U B=+\infty, x=0$ (zero initial investment in storage). Repeatedly solve the linear program (36) for each value of $u \in U=\left\{u^{(1)}, \ldots, u^{(m)}\right\}$ :

$$
\min _{y \in F(x, u)} d^{T} y
$$

Identify the scenario that results in the maximum objective value and initialize the set $\Theta$ with the index of this scenario.

2) Solve the following master problem:

$$
\min c^{T} x+\gamma
$$

subject to

$$
\begin{gathered}
\gamma \geq d^{T} y^{(s)}, s \in \Theta \\
A x \leq b, x \in\{0,1\} \\
G y^{(s)} \leq h, s \in \Theta \\
T x+Q y^{(s)} \leq r, s \in \Theta \\
I_{u} y^{(s)}=u^{(s)}, s \in \Theta
\end{gathered}
$$

Compute the optimal solution $\left(x^{*}, \gamma^{*},\left.y^{(s) *}\right|_{s \in \Theta}\right)$ and update $L B=c^{T} x^{*}+\gamma^{*}$.

3) Solve the slave problem (35): with $x$ set to $x^{*}$, solve the linear program (36) for each value of $u \in U$, and identify the scenario that results in the maximum objective value $\rho^{*}$; include the index of this scenario in set $\Theta$. Update $U B=\min \left(U B, c^{T} x^{*}+\rho^{*}\right)$.

4) If $(U B-L B) \leq \epsilon$, print the nonadjustable decision variables $x^{*}$ and terminate. Otherwise, go to step 2.

Step 2 in the above procedure generates new variables and constraints at every iteration, and gives the algorithm the name $\mathrm{C} \& \mathrm{CG}$; it is also known as the Benders primal-cutting plane algorithm [14] due to its similarity with the classical Benders decomposition (the Benders dual-cutting plane algorithm). Ref. [14] has shown that if the two-stage robust optimization problem is in the form (26)-(31) and the relatively complete recourse assumption holds, then the Benders primal-cutting plane algorithm terminates in a finite number of iterations and is significantly superior to the dual one in terms of convergence. In fact, Ref. [23] proposed speeding up the convergence of the Benders dual-cutting plane algorithm by adding scenarios corresponding to extreme points generated by the slave problem; these scenarios are similar to those used by the C\&CG algorithm, and further demonstrate their effectiveness in solving practical problems. The linear problems (36) in step 3 are similar to each other except for a change in the right hand side $u$ of the constraints; they can be solved efficiently by applying, for instance, the dual simplex method. It is important to note that if the penalty parameters for curtailment $\left(K_{D}\right.$ and $K_{R}$ ) are sufficiently large, then the adjustable variables result in a feasible system operation that does not entail power curtailment for any realization of uncertainty in $U$.

Although ROSION computes the design decision variables by considering the discrete uncertainty set $u \in U=$ $\left\{u^{(1)}, \ldots, u^{(m)}\right\}$, it also immunizes the system operation against all instances of uncertainty in the convex hull of $U$ : 
$u \in \operatorname{conv}(U)=\left\{\sum_{s=1}^{m} \beta_{s} u^{(s)} \mid\left(\beta_{s} \geq 0, s=1, \ldots m\right), \sum_{s=1}^{m} \beta_{s}=1\right\}$

This property is demonstrated in the appendix of the paper.

The second-stage problem with a discrete uncertainty set (in step 3) can be reformulated as an MILP problem with big-M constraints. In fact, exact MILP reformulations [14], [20], [21] and outer approximations [22], [23] have been developed primarily to handle the case when the uncertainty set is polyhedral. Both the exact and the approximate approaches that handle general polyhedral uncertainty sets are applicable to the storage planning problem because ROSION immunizes the system operation against all instances in the convex hull of the discrete uncertainty set $U$. Therefore, the second-stage problem can be solved by starting from the convex hull of $U$ (43), rather than the discrete set $U$ itself. In this case of polyhedral uncertainty, there are two approaches for solving the second-stage problem, both of which involve MILP reformulations with big-M constraints: (i) using KKT conditions and the relatively complete recourse assumption as described in [14], and (ii) using strong duality and the relatively complete recourse assumption that lead to a bilinear formulation as described in [20], [21] - c.f. (48)-(50) in the appendix. MILP approaches for solving the secondstage problem are known to be computationally intensive, and exactly solving the two-stage robust optimization problem with general polyhedral uncertainty sets remains challenging [14]; to speed up the process, Refs. [22], [23] proposed computing an approximate solution of the second-stage problem by using an outer approximation of the bilinear problem.

\section{NumericAl RESUlts}

ROSION was programmed in MATLAB and the MILP and LP programs were solved using CPLEX [26]. CPLEX was run with a relative optimality gap tolerance of $0.1 \%$ and the $\mathrm{C} \& \mathrm{CG}$ algorithm termination tolerance $\epsilon$ was set to $10^{-4}$. The simulations were carried out on an iMAC with $2.7 \mathrm{GHz}$ quadcore Intel Core i5 processor with 4MB L3 cache and $8 \mathrm{~GB}$ of RAM. The planning results are reported on the IEEE 14bus and the IEEE 118-bus networks whose original data sets are given with the distribution files of MATPOWER [27], and modified according to [28]. The convex quadratic generation cost curves were replaced with a four-segment linear approximation, where each segment approximates the generator quadratic cost curve over one fourth of its dispatch range. The IEEE 14-bus network has 2 wind farms connected at nodes 2 and 3 respectively, with each having an installed capacity of 40 MW; the IEEE 118-bus network has 5 wind farms connected at nodes $16,37,48,75$, and 83 , with a power capacity of 200 MW for each. The installed capacities translate into renewable energy penetration relative to the total peak load of $30.89 \%$ for the 14-bus network and $23.57 \%$ for the 118 -bus network. Each network was simulated over a period of 24 hours with each time slot spanning 1 hour. The scenarios that define the discrete uncertainty set ideally make use of forecasts that are based on historical observations together with economic predictions [16]-[18], and are constructed to represent extreme operating cases; however, they were simulated in this study using the data in [29] for load profiles and in [28] for wind profiles. A different hourly load profile was simulated for each of the 364 days (52 weeks $\times 7$ days/week) identified in [29]. The profile, expressed as a percentage of the annual peak demand in the original data set, was computed using specific multiplying coefficients for the week of the year (Table 2 in [29]), for the day of the week (Table 3 in [29]), and for the hour of the day (Table 4 in [29]). For each daily load profile, three wind generation profiles were simulated giving a discrete uncertainty set comprised of $3 \times 364=1092$ scenarios. The wind generation profiles were simulated by assuming that the wind speed $V$ is Weibull distributed with a scale factor of $11.0086 \mathrm{~m} / \mathrm{s}$ and a shape factor $1.9622 \mathrm{~m} / \mathrm{s}$; the corresponding output of the wind turbine generator is given by [28]:

$$
P_{W}= \begin{cases}0, & 0 \leq V<V_{c i} \\ P_{\text {rated }}\left(V-V_{c i}\right) /\left(V_{\text {rated }}-V_{c i}\right), & V_{c i} \leq V<V_{\text {rated }} \\ P_{\text {rated }}, & V_{\text {rated }} \leq V \leq V_{c o} \\ 0, & V>V_{c o}\end{cases}
$$

where the cut-in speed $V_{c i}=4 \mathrm{~m} / \mathrm{s}$, the rated speed $V_{\text {rated }}=$ $13.61 \mathrm{~m} / \mathrm{s}$, and the cut-out speed is $V_{c o}=25 \mathrm{~m} / \mathrm{s}$. The construction cost of storage devices in (1) was obtained from [6]: $\$ 160$ for each $\mathrm{kW}$ of rated power, $\$ 240$ for each $\mathrm{kWh}$ of rated energy, and $K_{S}=0.03$ (assuming a total planning horizon of 30 years). The available storage devices were considered to be in multiples of 32 MWh storage capacity, with the maximum charge/discharge rates per hour set at $25 \%$ of this capacity [13]. The efficiencies for charging and discharging were considered to be $90 \%$ each, giving an overall roundtrip efficiency of $81 \%$ [6]. All buses were assumed to be candidates for storage placement.

\section{A. IEEE 14-bus System}

Different ramp-rate limitations were considered for the conventional generators [1]; in particular, the generators were placed in two groups [6], one with high ramp rates allowing the generation to adjust over the intervals, and the other with low ramp rates requiring the generators to have the same dispatch over the intervals. For the IEEE 14-bus system, the slack generator at bus 1 is assumed to have an hourly ramp-rate of $45 \%$ of the generator's capacity; without any wind integration or storage investment, the adjustable variables produce a feasible solution free of load shed for each of the 364 load profiles. However, when the wind generation was installed without any investment in storage, instances of both renewable power and load curtailment were observed. Renewable power curtailment was observed in cases where wind power increased rapidly, load dropped, and the conventional generation could not ramp down to balance the system; load curtailment was observed when wind power dropped, load increased, and the conventional generation could not meet the load due to network flow or ramp-rate limitations. ROSION was used to install storage devices in five trials, each involving increased potential for storage capacity at a bus in increments 
of $32 \mathrm{MWh}: 32 \mathrm{MWh}, 64 \mathrm{MWh}, 96 \mathrm{MWh}, 128 \mathrm{MWh}$, and $160 \mathrm{MWh}$. Table I summarizes the planning results including the investment cost, the average daily cost of the dispatch from conventional generation over the 1092 scenarios, and the storage placement. The results show that the lowest investment cost is $\$ 71.68 \mathrm{M}$ with two storage units of capacity $128 \mathrm{MWh}$ at buses 3 and 5. Fig. 2 depicts the activity of these storage devices for five significant scenarios that contribute to the worst-case cost. For this system, the lowest average daily dispatch cost is observed in the case with five $64 \mathrm{MWh}$ storage devices that are installed at a cost of $\$ 89.6 \mathrm{M}$; the maximum difference in the average dispatch cost is less than $\$ 350$ per day or $0.45 \%$ of the minimum cost.

\section{B. IEEE 118-bus System}

The IEEE 118-bus system was simulated with more flexibility in the ramping capacity of its conventional generating units; the hourly ramp rates were set to $45 \%$ of the maximum generation for the largest units in the system, which are located at buses $8,69,80$ and 89 . However, in the absence of storage, scenarios with load shed were still observed in cases where wind power generation dropped quickly and the conventional generators did not have sufficient ramp-up capacity to meet the increasing load. The storage planning results in Table II show that there are three options that entail the same investment cost: eight $32 \mathrm{MWh}$ units, four $64 \mathrm{MWh}$ units, and two $128 \mathrm{MWh}$ units; the lowest average operational cost is for the $128 \mathrm{MWh}$ storage capacity concentrated at two buses. Out of the five cases, the last one with the highest investment cost of $\$ 89.6 \mathrm{M}$ yields the lowest average daily dispatch cost. The maximum difference in the average dispatch cost is however $\$ 224$, which is equivalent to around $0.02 \%$ of the minimum cost value.

In all test instances, no curtailment of load or renewable power appears for each of the 1092 scenarios; this is further confirmed for each of the planning solutions in Tables I and II via a Monte-Carlo simulation of 10,000 scenarios uniformly sampled from the convex hull of the discrete uncertainty set.

\section{Computational Performance}

Table III shows the computational performance of ROSION on the test instances in Tables I and II. The table summarizes the number of iterations, the time to solve the master problem, and the time to solve the slave. The number of iterations is relatively small, indicating that ROSION can rapidly identify scenarios that contribute to the worst case operation of the system; this behavior is particular to C\&CG algorithms and has been observed on other two-stage robust optimization problems [14]. In comparison, when CPLEX was applied directly to the complete two-stage robust model (26)-(31) with 1092 scenarios, it failed to find a feasible solution within a 5 hour time limit even for the 14-bus system.

\section{CONCLUSION}

This paper described a novel optimization tool, called Robust Optimization for Storage Investment on Networks ROSION, for storage planning under uncertainty. The uncertainty is modeled via a discrete set that is ideally obtained

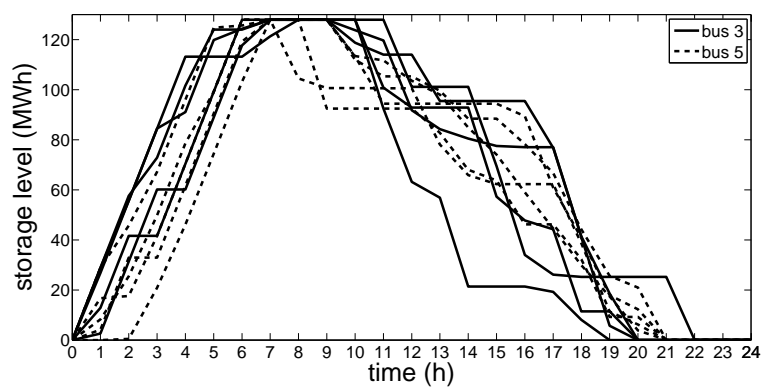

Fig. 2. Storage activity in the IEEE 14-bus network

Table I

Storage Planning For the IEEE 14-BUS NETwORK

\begin{tabular}{ccccc}
\hline $\begin{array}{l}\text { Capacity } \\
\text { (MWh) }\end{array}$ & Nb. & $\begin{array}{c}\text { Investment } \\
\text { Cost (M\$) }\end{array}$ & $\begin{array}{c}\text { Average } \\
\text { Dispatch } \\
\text { Cost (\$) }\end{array}$ & Location \\
\hline 32 & 10 & 89.60 & 77600 & $2,3,4,5,6$, \\
& & & $7,8,9,12,13$ \\
\hline 64 & 5 & 89.60 & 77430 & $3,4,5,6,8$ \\
\hline 96 & 3 & 80.64 & 77540 & $3,4,5$ \\
\hline 128 & 2 & 71.68 & 77779 & 3,5 \\
\hline 160 & 2 & 89.60 & 77476 & 3,5 \\
\hline
\end{tabular}

Table II

Storage Planning For the IEEE 118-BUS NETWORK

\begin{tabular}{ccccc}
\hline $\begin{array}{c}\text { Capacity } \\
(\mathbf{M W h})\end{array}$ & Nb. & $\begin{array}{c}\text { Investment } \\
\text { Cost }(\mathbf{M} \mathbf{\$})\end{array}$ & $\begin{array}{c}\text { Average } \\
\text { Dispatch } \\
\text { Cost } \mathbf{( \$ )}\end{array}$ & Location \\
\hline 32 & 8 & 71.68 & 1332922 & $\begin{array}{c}5,9,32,50, \\
59,67,94, \\
103\end{array}$ \\
\hline 64 & 4 & 71.68 & 1333025 & $\begin{array}{c}60,93,111, \\
115\end{array}$ \\
\hline 96 & 3 & 80.64 & 1332811 & $25,53,105$ \\
\hline 128 & 2 & 71.68 & 1332910 & 66,89 \\
\hline 160 & 2 & 89.60 & 1332801 & 59,82 \\
\hline
\end{tabular}

Table III

COMPUtATIONAl PeRformance of ROSION

\begin{tabular}{lccccc}
\hline System & $\begin{array}{c}\text { Capacity } \\
\text { (MWh) }\end{array}$ & Iter. & $\begin{array}{c}\text { Master } \\
\text { Time } \\
\text { (s) }\end{array}$ & $\begin{array}{c}\text { Slave } \\
\text { Time } \\
\text { (s) }\end{array}$ & $\begin{array}{c}\text { Total } \\
\text { Time } \\
\text { (s) }\end{array}$ \\
\hline \multirow{4}{*}{ 14-bus } & 32 & 3 & 6 & 277 & 283 \\
& 64 & 5 & 36 & 348 & 384 \\
& 96 & 6 & 80 & 346 & 426 \\
& 128 & 5 & 75 & 260 & 335 \\
& 160 & 5 & 61 & 247 & 308 \\
\hline \multirow{4}{*}{ 118-bus } & 32 & 3 & 313 & 2004 & 2317 \\
& 64 & 3 & 247 & 1778 & 2025 \\
& 96 & 3 & 439 & 1792 & 2231 \\
& 128 & 3 & 335 & 1669 & 2004 \\
& 160 & 2 & 77 & 1152 & 1229 \\
\hline
\end{tabular}


from historical observations and forecasts of coupled load and renewable generation profiles. ROSION computes a storage plan that allows satisfying the load and accepting the renewable power for all scenarios in the convex hull of the discrete uncertainty set; it is particularly useful in systems with limited ramping capabilities or congested lines. Unlike previous work, ROSION models the available storage device parameters, namely the rated power and energy, as varying in discrete quantities. In addition, the storage dynamics are modeled such that for the optimal storage placement and sizing, there will always exist a control action that can be implemented after the uncertainty is revealed and for which simultaneous charging and discharging does not occur. The computational engine behind ROSION is a column-and-constraint generation algorithm; its performance on the storage planning problem, formulated as a two-stage robust optimization program, shows that it can efficiently identify key scenarios that are sufficient to define the optimal design policy.

\section{APPENDIX}

This section demonstrates that ROSION immunizes the system operation against all instances in the convex hull of the discrete uncertainty set $U$. Let $\varphi, \lambda$, and $\nu$ denote the Lagrange multiplier vectors of $G y \leq h, Q y \leq r-T x$, and $I_{u} y=u$, respectively. The dual of problem (36) is the linear program:

$$
\max _{\varphi, \lambda, \nu}(T x-r)^{T} \lambda-h^{T} \varphi+u^{T} \nu
$$

subject to

$$
-Q^{T} \lambda-G^{T} \varphi+I_{u}^{T} \nu=d, \varphi \geq 0, \lambda \geq 0
$$

By combining (35) and (45)-(46), the slave problem (35) can be written in terms of the dual variables as:

$$
\max _{\varphi, \lambda, \nu, u}(T x-r)^{T} \lambda-h^{T} \varphi+u^{T} \nu
$$

subject to (46) and $u \in U$. Now substitute (43) in the dual slave objective function (47); this results in the following slave problem:

$$
\max _{\varphi, \lambda, \nu, \beta_{s}}(T x-r)^{T} \lambda-h^{T} \varphi+\sum_{s=1}^{m} \beta_{s}\left(u^{(s) T} \nu\right)
$$

subject to

$$
\begin{gathered}
-Q^{T} \lambda-G^{T} \varphi+I_{u}^{T} \nu=d, \varphi \geq 0, \lambda \geq 0 \\
\sum_{s=1}^{m} \beta_{s}=1 ; \beta_{s} \geq 0, s=1, \ldots m
\end{gathered}
$$

Given that $\beta_{s}$ and $\nu$ do not appear in the same constraint equations (49)-(50), it is evident that $\beta_{s}(s=1, \ldots, m)$ can be chosen independently of other variables such that $\sum_{s=1}^{m} \beta_{s}\left(u^{(s) T} \nu\right)$ attains its maximum value; an optimal choice is $\beta_{s}=1$ for the scenario $s$ with maximum $\left(u^{(s) T} \nu\right)$. Therefore, the solution of the dual slave problem with the uncertainty set given by (43) can be obtained by solving (48)-(50) $m$ times, where $\beta_{s}=1$ is considered separately for $s=1, \ldots, m$. This is the same as the solution of the dual problem (45)-(46) that gives the maximum objective function value over the discrete uncertainty set $U$, which is also equivalent to the solution of the primal slave problem in steps 1 and 3 in ROSION (due to strong duality and the relatively complete recourse assumption). In other words, the solution of the two-stage robust storage investment problem with the uncertainty set (43) can be obtained using exactly the same steps in ROSION.

\section{REFERENCES}

[1] B. Hartmann and A. Dán, "Methodologies for storage size determination for the integration of wind power," IEEE Trans. Sustain. Energy, vol. 5, no. 1, pp. 182-189, Jan. 2014.

[2] K. Dvijotham, S. Backhaus, and M. Chertkov, "Operations-based planning for placement and sizing of energy storage in a grid with a high penetration of renewables," http://arxiv.org/abs/1107.1382v2, Jul. 2011.

[3] K. M. Chandy, S. H. Low, U. Topcu, and H. Xu, "A simple optimal power flow model with energy storage," in 49th IEEE Conference on Decision and Control (CDC), 15-17 Dec. 2010, pp. 1051-1057.

[4] S. Carr, G. C. Premier, R. M. Dinsdale, A. J. Guwy, and J. Maddy, "Energy storage for active network management on electricity distribution networks with wind power," in International Conference on Renewable Energies and Power Quality (ICREPQ'12), 28-30 Mar. 2012, pp. 1-6.

[5] E. Sjödin, D. F. Gayme, and U. Topcu, "Risk-mitigated optimal power flow for wind powered grids," in 2012 American Control Conference (ACC), 27-29 Jun. 2012, pp. 4431-4437.

[6] H. Oh, "Optimal planning to include storage devices in power systems," IEEE Trans. Power Syst., vol. 26, no. 3, pp. 1118-1128, Aug. 2011.

[7] M. Ghofrani, A. Arabali, M. Etezadi-Amoli, and M. S. Fadali, "A framework for optimal placement of energy storage units within a power system with high wind penetration," IEEE Trans. Sustain. Energy, vol. 4, no. 2, pp. 434-442, Apr. 2013.

[8] T. K. A. Brekken, A. Yokochi, A. von Jouanne, Z. Z. Yen, H. M. Hapke, and D. A. Halamay, "Optimal energy storage sizing and control for wind power applications," IEEE Trans. Sustain. Energy, vol. 2, no. 1, pp. 6977, Jan. 2011.

[9] Y. M. Atwa and E. F. El-Saadany, "Optimal allocation of ESS in distribution systems with a high penetration of wind energy," IEEE Trans. Power Syst., vol. 25, no. 4, pp. 1815-1822, Nov. 2010.

[10] A. Gabash and P. Li, "Active-reactive optimal power flow in distribution networks with embedded generation and battery storage," IEEE Trans. Power Syst., vol. 27, no. 4, pp. 2026-2035, Nov. 2012.

[11] — "Flexible optimal operation of battery storage systems for energy supply networks," IEEE Trans. Power Syst., vol. 28, no. 3, pp. 27882797, Aug. 2013.

[12] D. Gayme and U. Topcu, "Optimal power flow with distributed energy storage dynamics," in 2011 American Control Conference (ACC), 29 Jun.-1 Jul. 2011, pp. 1536-1542.

[13] — "Optimal power flow with large-scale storage integration," IEEE Trans. Power Syst., vol. 28, no. 2, pp. 709-717, May 2013.

[14] B. Zeng and L. Zhao, "Solving two-stage robust optimization problems using a column-and-constraint generation method," Operations Research Letters, vol. 41, no. 5, pp. 457-461, Sep. 2013.

[15] J. M. Mulvey, R. J. Vanderbei, and S. A. Zenios, "Robust optimization of large-scale systems," Operations Research, vol. 43, no. 2, pp. 264-281, Mar.-Apr. 1995.

[16] BC Hydro, "Electric Load Forecast: Fiscal 2013 to Fiscal 2033," Dec. 2012.

[17] Manitoba Hydro, "2013 Electric Load Forecast,” Jul. 2013.

[18] P. H. Henault, R. B. Eastvedt, J. Peschon, and L. P. Hajdu, "Power system long-term planning in the presence of uncertainty," IEEE Trans. Power Appar. Syst., vol. PAS-89, no. 1, pp. 156-164, Jan. 1970.

[19] B. Chen, J. Wang, L. Wang, Y. He, and Z. Wang, "Robust optimization for transmission expansion planning: Minimax cost vs. minimax regret," IEEE Trans. Power Syst., (available via Early Access) 2014.

[20] A. Thiele, T. Terry, and M. Epelman, "Robust linear optimization with recourse," Optimization Online, http://www.optimization-online. org/DB_FILE/2009/03/2263.pdf, Mar. 2010.

[21] L. Zhao and B. Zeng, "Robust unit commitment problem with demand response and wind energy," in 2012 IEEE Power and Energy Society General Meeting, Jul. 2012, pp. 1-8.

[22] R. Jiang, J. Wang, and Y. Guan, "Robust unit commitment with wind power and pumped storage hydro," IEEE Trans. Power Syst., vol. 27, no. 2, pp. 800-810, May 2012. 
[23] D. Bertsimas, E. Litvinov, X. A. Sun, J. Zhao, and T. Zheng, "Adaptive robust optimization for the security constrained unit commitment problem," IEEE Trans. Power Syst., vol. 28, no. 1, pp. 52-63, Feb. 2013.

[24] M. Kraning, Y. Wang, E. Akuiyibo, and S. Boyd, "Operation and configuration of a storage portfolio via convex optimization," in 18th International Federation of Automatic Control (IFAC) World Congress, 28 Aug.-2 Sep. 2011, pp. 10487-10 492.

[25] H. A. Taha, Operations Research: An Introduction - 5th Ed. Upper Saddle River, NJ: Prentice Hall, 1992.

[26] “IBM ILOG CPLEX. ver. 12.3," http://www-01.ibm.com/software/ integration/optimization/cplex-optimizer.

[27] R. D. Zimmerman, C. E. Murillo-Sánchez, and R. J. Thomas, "MAT POWER: Steady-state operations, planning, and analysis tools for power systems research and education,' IEEE Trans. Power Syst., vol. 26, no. 1, pp. 12-19, Feb. 2011.

[28] H. Yu and W. Rosehart, "An optimal power flow algorithm to achieve robust operation considering load and renewable generation uncertainties," IEEE Trans. Power Syst., vol. 27, no. 4, pp. 1808-1817, Nov. 2012.

[29] C. Grigg, P. Wong, P. Albrecht, R. Allan, M. Bhavaraju, R. Billinton, Q. Chen, C. Fong, S. Haddad, S. Kuruganty, W. Li, R. Mukerji, D. Patton, N. Rau, D. Reppen, A. Schneider, M. Shahidehpour, and C. Singh, "The IEEE reliability test system-1996. A report prepared by the reliability test system task force of the application of probability methods subcommittee," IEEE Trans. Power Syst., vol. 14, no. 3, pp. 1010-1020, Aug. 1999.

Rabih Jabr (M'02-SM'09) was born in Lebanon. He received the B.E. degree in electrical engineering (with high distinction) from the American University of Beirut, Beirut, Lebanon, in 1997 and the Ph.D. degree in electrical engineering from Imperial College London, London, U.K., in 2000. Currently, he is an Associate Professor in the Department of Electrical and Computer Engineering at the American University of Beirut. His research interests are in mathematical optimization techniques and power system analysis and computing.

Izudin Džafić (M'05-SM'13) received his Ph.D. degree from University of Zagreb, Croatia in 2002. He is currently an Associate Professor in the Department of Electrical Engineering at the International University of Sarajevo, Bosnia. From 2002 to 2014, he was with Siemens AG, Nuremberg, Germany, where he held the position of the Head of the Department and Chief Product Owner (CPO) for Distribution Network Analysis (DNA) R\&D. His research interests include power system modeling, development and application of fast computing to power systems simulations. Dr. Džafić is a member of the IEEE Power and Energy Society and the IEEE Computer Society.

Bikash C. Pal (M'00,SM'02,F'13) received the B.E.E. (with honors) degree from Jadavpur University, Calcutta, India, the M.E. degree from the Indian Institute of Science, Bangalore, India, and the Ph.D. degree from Imperial College London, London, U.K., in 1990, 1992, and 1999, respectively, all in electrical engineering. Currently, he is a Professor in the Department of Electrical and Electronic Engineering, Imperial College London. His current research interests include state estimation, power system dynamics, and flexible ac transmission system controllers. 